Rev. Adv. Mater. Sci. 57 (2018) 11-25

\title{
MAGNETOMETRIC STUDY OF ZnO/CoO NANOCOMPOSITES
}

\author{
J. Typek ${ }^{1}$, N. Guskos' ${ }^{1}$ G. Zolnierkiewicz'1, D. Sibera² and U. Narkiewicz² \\ ${ }^{1}$ Institute of Physics, West Pomeranian University of Technology, Al. Piastow 48, 70-311 Szczecin, Poland \\ ${ }^{2}$ Institute of Chemical and Environment Engineering, Faculty of Chemical Technology and Engineering, \\ West Pomeranian University of Technology, Pulaskiego 10, 70-322 Szczecin, Poland
}

Received: January 06,2018

\begin{abstract}
ZnO nanoparticles doped with transition metal ions are intensively studied nanomaterials, due to their charges and the spins of electrons that provides new magnetic, optical and transport properties. They find a vast range of applications, ranging from optoelectronics to spintronics. In this context especially important is the room temperature ferromagnetism observed for $\mathrm{ZnO}$ doped nanomaterials, although this phenomenon is still a controversial and open topic in material science, mostly due to low reproducibility of results from samples prepared by different techniques. In the first part of this article a short review of papers using magnetometric methods to determine the magnetic characteristics of Co-doped $\mathrm{ZnO}$ nanomaterials is presented. Different models introduced to explain room temperature ferromagnetism (carrier mediated ferromagnetism, $\mathrm{Co}^{2+}$-oxygen vacancy pairs, blocked superparamagnetic clusters, $\mathrm{Co}^{2+} \mathrm{Zn}$ interstitial pairs, heterogeneous distribution of magnetic ions) are examined and discussed. In the second part, magnetisation study of a new series of $n \mathrm{CoO} /(1-n) \mathrm{ZnO}$ nanocomposites synthesized by hydrothermal method under higher than previously applied pressure will be described. The obtained experimental results will be analysed and information on magnetic systems responsible for the observed characteristics and the involved magnetic interactions will be deduced.
\end{abstract}

\section{INTRODUCTION}

Among various types of nanoparticles reported in the literature, e.g. metal nanoparticles, metal oxide nanoparticles, and polymer nanoparticles, zinc oxide $(\mathrm{ZnO})$ nanoparticles have their own importance due to a vast range of applications [1-3]. $\mathrm{ZnO}$ is an promising material for short-wavelength optoelectronic applications owing to its wide band gap $3.37 \mathrm{eV}$, large bond strength, and large exciton binding energy (60 meV) at room temperature (RT) [4]. As a wide band gap material, $\mathrm{ZnO}$ is used in solid state blue to ultraviolet (UV) optoelectronics, including laser applications. $\mathrm{ZnO}$ nanoparticles also found uses in solar cells, photocatalytic and photoluminescence devices, as gas sensors, chemical and biosensors, light emitting diodes, and photodetectors [5]. Moreover, due to its noncentrosymmetric crystallographic phase, $\mathrm{ZnO}$ shows the piezoelectric properties, which are highly valuable for the fabrication of electromagnetic coupled sensors and actuators [6].

Various synthetic methods to grow a variety of $\mathrm{ZnO}$ nanostructures have been used, including nanoparticles, nanowires, nanorods, nanotubes, nanobelts, and other complex morphologies [7-9]. The sol-gel method (solution method) or the hydrothermal method is the most often used because of its ease of handling, reliability, repeatability, and environmental friendliness. In addition, synthesis of $\mathrm{ZnO}$ nanoparticles in the solution provides well-defined shapes and sizes of zinc oxide nanoparticles.

Corresponding author: J. Typek, e-mail: Janusz.Typek@zut.edu.pl 
It has been shown that solvent has an important effect on the morphology of the final $\mathrm{ZnO}$ nanomaterial. As various applications depend crucially upon properties such as nanoparticle size, its dispersity, shape, surface state, crystal structure, agglomeration state, they must be controlled by tuning different parameters during the synthesis process, e.g., the precursor type and its concentration, types of capping molecules, kinds of solvent, reaction time, and reaction temperature [10].

Doping of $\mathrm{ZnO}$ nanoparticles with transition metal ions further increases their application potential due to the charges and the spins of electrons that provides new magnetic, optical and transport properties of these materials [11-14]. Especially important is the room temperature ferromagnetism (RTFM), observed in in V-, Mn-, Fe-, Co-, Ni-, and $\mathrm{Cu}$-doped $\mathrm{ZnO}$, although reports of detected ferromagnetic (FM) properties are inconsistent [15]. This might be the result of sensitivity of this phenomenon to the preparation method. Thus RTFM may arises from precipitation of the secondary phases and not from the replacement of $\mathrm{Zn}$ ion by the transition metal ion in $\mathrm{ZnO}$ lattice. On the other hand, theoretical study suggested that oxygen vacancies can make a significant contribution to FM by forming bound magnetic polaron [16]. Consequently, the presence of FM in transition metal doped $\mathrm{ZnO}$ is still an open topic in material science, mostly due to low reproducibility of results from samples prepared by different techniques.

In the first part of this article a short review of papers using magnetometric methods to determine the magnetic characteristics of Co-doped $\mathrm{ZnO}$ nanomaterials will be presented. Five main groups of hypotheses (carrier mediated ferromagnetism, $\mathrm{Co}^{2+}$-oxygen vacancy pairs, blocked superparamagnetic clusters, $\mathrm{Co}^{2+}-\mathrm{Zn}$ interstitial pairs, heterogeneous distribution of magnetic ions) will be examined and discussed. Influence of preparation methods used in synthesis of the discussed materials and structural characteristic on their magnetic properties will be briefly described. Recently, a new series of $n \mathrm{CoO} /(1-n) \mathrm{ZnO}$ nanocomposites synthesized by hydrothermal method under higher than previously applied pressure was carried out [17]. It allowed to achieve more concentrated Co nanocomposites (up to $n=0.7$ ). DC magnetisation study of these samples will be described in the second part of this paper. Magnetic susceptibility measurements in zero field cooled (ZFC) and field cooled (FC) modes in 2 - 290K temperature range as well as isothermal magnetisation in an external magnetic field up to $70 \mathrm{kOe}$ will be analysed. From the obtained experimental results the information on magnetic systems responsible for the observed characteristics and the involved magnetic interactions will be discussed.

\section{SHORT REVIEW OF PAPERS ON MAGNETIC PROPERTIES OF $\mathrm{NCoO} /(1-\mathrm{N}) \mathrm{ZnO}$ NANOCOMPOSITES.}

\subsection{Magnetism of Co doped of $\mathrm{ZnO}$}

Solubility limit. Magnetic properties of Co doped $\mathrm{ZnO}$ nanoparticles strongly depend on concentration of this magnetic ion in the zinc oxide matrix in regard to solubility limit. The reported values of solubility limit of $\mathrm{Co}$ in $\mathrm{ZnO}$ vary in a broad range, depending mostly on sample preparation method [1822]. For many transition metal ions in the diluted magnetic oxides this limit is usually low. For Codoped bulk $\mathrm{ZnO}$ it is close to 10 at.\% [17]. The highest solubility limit show samples prepared by the pulsed laser deposition or ion implantation method. A very high value of 40 at. $\%$ was reported for films prepared by pulsed laser deposition technique [19]. In contrast, a strong phase separation and formation of $\mathrm{Co}_{3} \mathrm{O}_{4}$ was observed for films prepared by the meta-organic deposition with 5 at.\% of cobalt [20]. In nanoalloys the solubility limit of 15 at.\% was observed [21], while for samples prepared by sol-gel and RF sputtering it is close to 12 at $\%$ [22]. It was shown that grain boundaries and free surfaces drastically shifts the solubility limit in zinc oxide towards higher concentrations of magnetic ions [23]. Thus the solubility of cobalt in zinc oxide increases considerably with diminishing grain size. The solubility limit of cobalt in thin zinc oxide films is 33 at. \% of $\mathrm{Co}$ at $550^{\circ} \mathrm{C}$ (the synthesis temperature of the films), although the total solubility of cobalt does not exceed 2 at.\% of Co in the bulk of zinc oxide [23]. Above 33 at.\% of Co limit the second phase, $\mathrm{Co}_{2} \mathrm{O}_{3}$ with the cubic lattice appears and the period of the wurtzite zinc oxide lattice ceases to increase as the cobalt concentration rises [23]. RTFM in undoped ZnO. Discussing RTFM in Co doped $\mathrm{ZnO}$, it must be acknowledged that FM was also reported in undoped $\mathrm{ZnO}$ nanomaterial [24]. In order to provide magnetic moments, deviations from the empty or filled shell electron configuration must be present. Tietze et al. have put forward a hypothesis that FM in undoped $\mathrm{ZnO}$ stems from magnetic moments located in the grain boundaries [25]. Using a low energy muon spin relaxation in combination with SQUID and TEM techniques, they demon- 
strated that the magnetic volume fraction is strongly related to the sample volume fraction occupied by grain boundaries. By means of electronic structure theory (Density Functional Theory) they proved that the formation of unpaired electrons is possible at grain-boundaries and that geometries may exist, where such electrons are coupled ferromagnetically [25].

Another possible mechanism of RTFM in undoped $\mathrm{ZnO}$ was put forward by Zhang et al. to describe this phenomenon in the undoped polycrystalline $\mathrm{ZnO}$ thin films fabricated under different oxygen partial pressures [26]. Magnetism is restricted to some defect-rich regions, such as the surface, interface, and grain boundary, and is not uniform throughout the samples. Zhang et al. have explained their experimental results by putting forward a model with interstitial $\mathrm{Zn}$ ions [26]. The shallow donors caused by these defects might modify the electronic structure of undoped $\mathrm{ZnO}$ thin films, leading to the RT FM. Interestingly, in other studies with undoped $\mathrm{ZnO}$ nanoparticles it was shown that even absorption of certain organic molecules on nanoparticle surface can induce FM-like behaviour [27].

Carrier mediated ferromagnetism. From the early theoretical works at the beginning of XXI century it is expected that the goal of obtaining a controllable RT semiconducting ferromagnet, which could be used in spintronic devices, could be accomplished by incorporation of transition metal or rare earth ions into nonmagnetic semiconductor host lattice [28,29]. Obtained in this way a diluted magnetic semiconductor is predicted to raise Curie temperature above RT. The crucial role in formation of RTFM is played by charge carriers in the form of holes or electrons. In $\mathrm{ZnO}$ :Co the coupling between localised magnetic Co $3 d$ states is achieved through mediation of $O p$ orbitals (superexchange interaction). Reducing conditions in the form of addition of $\mathrm{Zn}$ vapour or low $\mathrm{O}_{2}$ partial pressure (intrinsic doping ) or extrinsic n-type doping (e.g. Al) are needed for the creation of FM state [30]. The threshold for the onset of FM would be the addition of 0.5 electron to the system per one Co ion, while a maximum in the FM interaction could reached at the addition of about one electron per one Co ion [30].

$\mathrm{Co}^{2+}$ oxygen vacancy pairs. Pemmaraju et al. have shown, using Density Functional Theory calculations, that RTFM in Co doped $\mathrm{ZnO}$ may originate from $\mathrm{Co}^{2+}$ oxygen-vacancy pairs with a partially filled level close to the $\mathrm{ZnO}$ conduction band minimum [31]. Calculations has proved that oxygen vacancy
$\left(V_{o}\right)$ concentration in $\mathrm{ZnO}$ will be close to $1 \%$ and they will be close to $\mathrm{Co}^{2+}$ ions. The magnetic interaction between these pairs is sufficiently long-ranged to cause percolation at moderate concentrations. The presence of additional electron donors greatly increases this interaction [31]. Similar results were reported by Tao et al. [16]. They have studied effects of $V_{O}$ on magnetic properties of Co-doped $\mathrm{ZnO}$ by first principle calculations. The calculated formation energies of $V_{O}$ with different bonding to Co atom indicate that $V o$ prefers location near $C o$ atom, implying a strong local interaction between the Co atom and oxygen vacancy. Induced by Vo, the Co$3 d$ and O-2p valence bands upward shift towards the Fermi level, leads to the presence of additional carriers at the Fermi level.

The delocalized carriers and the substitutional Co ion play a key role in the occurrence and stability of FM of Co-doped $\mathrm{ZnO}$ [16]. Experimental studies on $\mathrm{Zn}_{1-x} \mathrm{Co}_{x} \mathrm{O}(x=0.002,0.004,0.006$, and 0.008$)$ nanoparticles synthesized by a sol-gel process has shown a crucial role of the lattice defects due to oxygen vacancies [32]. The observed long-range FM ordering was explained by using the bond magnetic polaron model (BMPM), first introduced by Coey et al. [33]. A BMPM was introduced for insulating systems and constitutes of a charge carrier localized on oxygen vacancy interacting with magnetic metal cation. Magnetic polarons interact ferromagnetically. When concentration of magnetic polarons reaches a percolation limit, long-range FM interaction arises leading to ferromagnetic nature of these solids. There are very promising objects in spintronics as the control of carrier concentration enables easy manipulation of their magnetic properties.

Blocked superparamagnetic clusters. Usually, RTFM is inferred from SQUID magnetisation studies and the presence of magnetic hysteresis is regarded as sufficient evidence of FM state. Hanafin et al. has noticed that large blocked superparamagnetic clusters may mimic FM response [34]. They have introduced a model containing two different Co-related magnetic centres: $\mathrm{Co}^{2+}$ and $\mathrm{Co}^{2+}-V_{O}$. The two-centre model percolates for $\mathrm{Co}^{2+}-V_{O}$ concentration of about $7 \%$ what is quite large, but if blocked superparamagnetic clusters are taken into account it may explain RTFM. Clusters containing few hundreds of magnetic ions should be blocked at RT. It was shown by computer simulation of two-spin correlations that there are enough clusters surviving at high temperatures to consider the model as realistic in explaining RTFM in Co doped $\mathrm{ZnO}$ [34]. 
Ney et al. have investigated Co-doped ZnO films epitaxially grown on sapphire by reactive magnetron sputtering [35]. For optimized growth conditions virtually all Co dopant atoms were located on substitutional Zn lattice sites. Magnetometric studies showed that the samples behaved as a Brillouinlike paramagnet with $S=3 / 2$ and $L=1$. Reducing the oxygen content during preparation has led to the onset of phase separation. Such samples behaved like a blocked superparamagnetic ensemble. In the entire range of preparation conditions no signs of intrinsic FM were found [35].

An exotic model of RTFM, based on Lieb-Mattis theorem, was proposed by Kuzian et al. [36]. Lieb and Mattis have showed that the ground state of an AFM system depends on the topology of the interacting bonds and, under certain conditions, it is FM rather than AFM. Kuzmian et al. have studied various structures formed by the interacting magnetic impurities (e.g. Co) in wurtzite semiconductors. They tookAFM nearest-neighbour interaction into account. Firstly, they constructed several finite clusters that showed an enhanced magnetic response at low temperatures and below a certain temperature their magnetic susceptibility exceeded that of non-interacting spins (isotropic superparamagnetic response). Thus, an AFM interaction may result in an enhancement of magnetic response if the geometry of spin arrangement favours the formation of a ferrimagnetic ground state. In the following step they constructed extended lattices of these clusters, which experienced ferromagnetic ordering transition at a finite temperature. The average ground-state spin per magnetic ion of spin $S$ tends to a finite value (of about $S=3$ ) despite a low concentration of magnetic ions [36].

Co ${ }^{2+}-Z n$ interstitial pairs. Shah et al. have demonstrated that the BMPM is responsible for FM in Co$\mathrm{ZnO}$ semiconductors, where the carriers are provided by the interstitial zinc $\left(\mathrm{Zn}_{\mathrm{i}}\right)$ [37]. Each interstitial is regarded as a donor defect site. The defect site and an electron associated with it form a hydrogenic atom. In such a system, when the donor concentration increases, the $1 \mathrm{~s}$ orbitals of the hydrogenic atoms start to overlap and an impurity band forms. This impurity band overlaps with the dopant states and this leads to FM exchange coupling. The percolation phenomenon occurs when these bond magnetic polarons roughly fill $16 \%$ of the space, resulting in the long-range FM order. Investigation of the structural, magnetic and transport properties of bulk and film samples gave consistent results in the context of the proposed tworegion model, i.e. (1) a $\mathrm{Zn}_{\mathrm{i}}$ layer where carriers are sufficient to couple Co ions ferromagnetically and (2) a region with little carriers that remain in a paramagnetic state [37].

Magnetic measurements on Co-doped ZnO samples $\mathrm{Co}_{y} \mathrm{Zn}_{1-y} \mathrm{O}$ prepared by magnetron sputtering done by Shi et al. have shown HTFM in samples with $y \leq 0.05$ [38]. An experimental and numerical study of the X-ray absorption near-edge structure spectra revealed that the incorporation of the $\mathrm{Zn}$ interstitials in the lattice is crucial to the appearance of RTFM in these samples.[38]. The influence of $Z n$ interstitials $\left(Z n_{i}\right)$ on physical properties of $C_{0}$ doped ZnO films was studied by Schwartz and Gamelin [39]. They demonstrated the first reversible $300 \mathrm{~K}$ FM ordering in a diluted magnetic semiconductor, achieved by lattice incorporation and removal of the native $n$-type $Z n_{i}$. Spectroscopic and magnetic data implicate a double-exchange mechanism for FM that involves electron delocalization among substitutionally doped cobalt ions [39].

Heterogeneous distribution of magnetic ions. There are three possible origins of RTFM in Co doped $\mathrm{ZnO}$ due to non-homogeneous distribution of magnetic ions: metal Co inclusions, intermetallic FM CoZn phases, and uncompensated spins on the surface of AFM nanocrystals [40].

The presence of metal $\mathrm{Co}$ inclusions has been detected e.g. by TEM, XRD and magnetic circular dichroism methods. Nay et al. have reported XAS and SQUID study of a comprehensive set of $\mathrm{Co}: \mathrm{ZnO}$ epitaxial thin film samples fabricated using three deposition methods [41]. Contrary to numerous claims in the literature, phase-pure, crystallographically excellent $\mathrm{Co}$ doped $\mathrm{ZnO}$ is uniformly PM, irrespective of the preparation method. FM-like behaviour is observed only for samples showing extensive defect formation or phase separation. Opel et al. have used X-ray magnetic circular dichroism measurements on the epitaxial $\mathrm{Zn}_{0.95} \mathrm{Co}_{0.05} \mathrm{O}$ thin films deposited on single crystalline, c-axis oriented $\mathrm{ZnO}(0001)$ substrates [42]. The obtained data provided clear evidence that the observed RTFM is not related to a bulk homogeneous material, but could rather be explained by the presence of superparamagnetic metallic cobalt precipitates. The presence of crystallographically oriented Co nanocrystal in $\mathrm{ZnO}$ matrix formed by ion implantation and postannealing has been found by Zhou et al. [43]. They established that certain magnetic properties of their samples, e.g., the anisotropy and the superparamagnetic blocking temperature, can be tuned by annealing [43]. Additionally, Kaspar et al. analysis of X-ray photoelectron spectroscopy depth profiling indicated the presence of $\mathrm{Co}(0)$ in 
the $\mathrm{Zn}$-treated films. Besides, they utilized X-ray absorption fine structure to identify the secondary phase as FM CoZn [44].

Finally, from magnetisation and spin-dependent magnetoresistance measurements Dietl et al. have argued that spontaneous magnetization arises from uncompensated spins at the surface of AFM nanocrystals of Co-rich wurtzite $(\mathrm{Zn}, \mathrm{Co}) \mathrm{O}$ [45]. Moreover, this model explains a large anisotropy observed in both magnetisation and magnetoresistance in terms of spin Hamiltonian of Co ions in the crystal field of the wurtzite lattice. Blasco et al. have investigated $\mathrm{Zn}_{0.96} \mathrm{Co}_{0.04} \mathrm{O}$ samples using different synthetic routes and found that the magnetic impurity corresponds to Co particles that can be formed in reducing conditions (low-oxygen pressure) [46]. Non-optimal synthetic conditions (oxidizing atmosphere or low temperature) have led to the formation of binary oxides $\left(\mathrm{CoO}\right.$ or $\left.\mathrm{Co}_{3} \mathrm{O}_{4}\right)$ and the samples exhibited only paramagnetic behaviour.

\subsection{Heavy Co doping of $\mathrm{ZnO}$}

Two papers by Kuryliszyn-Kudelska et al. [47,48] presented structural (XRD) and magnetic properties (determined by AC magnetic susceptibility measurements and DC SQUID magnetometry) of Co doped $\mathrm{ZnO}$ nanocrystals prepared by two methods: the microwave assisted hydrothermal synthesis (hydrothermal method) and traditional wet chemistry method followed by calcination (calcination method). Two series of samples in a wide range of $\mathrm{CoO}$ nominal concentration were obtained: from $5 \mathrm{wt} . \%$ to 80 wt. \% in the calcination method and from $5 \mathrm{wt} . \%$ to $60 \mathrm{wt} . \%$ in the hydrothermal method. The mean crystalline size of magnetic phases measured by XRD was from 14 to $55 \mathrm{~nm}$ for $\mathrm{Co}_{3} \mathrm{O}_{4}$ phases in samples obtained by the calcination method, while for samples obtained by the hydrothermal method it varied from 33 to $77 \mathrm{~nm}$ for $\mathrm{ZnCo}_{2} \mathrm{O}_{4}$ phases. For $\mathrm{ZnO}$ phases the average sizes varied from 64 to $300 \mathrm{~nm}$ (the higher the $\mathrm{CoO}$ content the larger the size of $\mathrm{ZnO}$ nanoparticles) [49]. The obtained results indicated that in both types of samples (hydrothermal or calcined) the crystalline size of the magnetic phases $\left(\mathrm{Co}_{3} \mathrm{O}_{4}\right.$ and $\left.\mathrm{ZnCo}_{2} \mathrm{O}_{4}\right)$ did not show monotonous dependence. The crystalline size of $\mathrm{ZnO}$ decreased with the increase of nominal concentration of $\mathrm{CoO}$ for samples obtained by calcination process, while for the second method of synthesis the opposite effect was observed. From scanning electron microscopy two morphologies could be distinguished: spherical and hexagonal nanograins were observe. The hexagonal nanocrystals were assumed to correspond to the $\mathrm{ZnO}$ phase, while the spheroidal nanocrystals to the spinel phases $\left(\mathrm{Co}_{3} \mathrm{O}_{4}\right.$ for samples produced by the calcination process and $\mathrm{ZnCo}_{2} \mathrm{O}_{4}$ for the hydrothermal method). Additionally, these measurements showed that in samples synthesized by the hydrothermal method the tendency to the formation of agglomerates is smaller in comparison to the other samples.

As the magnetic study of these samples has shown, two different types of magnetic behaviour was observed: spin-glasslike behaviour or superparamagnetic behaviour, depending on the synthesis processes of samples. In the high temperature range the Curie-Weiss behaviour was registered in AC magnetic susceptibility. It was observed that the determined negative value of the Curie-Weiss temperature $T_{0}$ depended strongly on the nominal content of cobalt oxide. It was revealed that for samples synthesized by the calcination method the values of $T_{0}$ increased with the increase of Co content indicating enhancement of AFM interaction. On the other hand, for samples obtained by the hydrothermal method the opposite effect was detected indicating the breakdown of the predominance of AFM coupling with the increase of nominal Co ion content. For sample produced by hydrothermal method having a low $\mathrm{CoO}$ content (5 wt.\%) a peak in the temperature dependence of real part of AC susceptibility around $40 \mathrm{~K}$ was registered. Such a feature is usually encountered in superparamagnetic and spin-glass systems. Measurements of the shift of this maximum towards higher temperatures with increasing driving frequency indicate that in this sample superparamagnetic system is realized.

Hadžić et al. have investigated $n \mathrm{CoO} /(1-n) \mathrm{ZnO}$ hydrothermal samples by Raman spectroscopy $[49,50]$. In the Raman spectra the presence of $\mathrm{ZnO}$ was established by the existence of characteristic single and multi-phonon modes. The presence of $\mathrm{ZnCo}_{2} \mathrm{O}_{4}$ phase was determined by the existence of its typical phonon modes. As consequence of small sizes of investigated particles, Raman peaks of registered phases were shifted and broadened compared to the bulk modes. Besides the modes that belong to $\mathrm{ZnCo}_{2} \mathrm{O}_{4}$ and $\mathrm{ZnO}$ phases, the presence of surface optical phonons modes was established. Investigation of the intensities of these modes showed that the intensity of $\mathrm{ZnO}$ modes decreased with the increase in $\mathrm{CoO}$ concentration, while the intensity of $\mathrm{ZnCo}_{2} \mathrm{O}_{4}$ modes displayed the opposite behaviour. 
Typek et al. have made measurements of magnetic resonance spectra and discussed magnetic properties of $n \mathrm{CoO} /(1-n) \mathrm{ZnO}$ hydrothermal samples with the composition index $n=0.4,0.5,0.6$, and 0.7 [17]. Three main features were revealed in the registered spectra - a broad line due mostly to $\mathrm{ZnCo}_{2} \mathrm{O}_{4}$ agglomerated nanoparticles, two narrow lines attributed to isolated, but interacting $\mathrm{Co}^{2+}$ ions in $\mathrm{ZnO}$ phase, and one narrow, asymmetric line, probably arising from $\mathrm{Co}_{3} \mathrm{O}_{4}$ nanoparticles. Notwithstanding heavy Co doping, the isolated $\mathrm{Co}^{2+}$ ions in $\mathrm{ZnO}$ phase were present as in a lightly doped $\mathrm{ZnO}$ bulk crystals. The dominating phase in the highly Co-doped $\mathrm{ZnO}$ nanoparticles was $\mathrm{ZnCo}_{2} \mathrm{O}_{4}$, but as the nanoparticles were strongly agglomerated, no superparamagnetic phase was observed in the high temperature range [17].

Two abundant secondary phases which appear in heavily Co doped $\mathrm{ZnO}$ samples are $\mathrm{Co}_{3} \mathrm{O}_{4}$ and $\mathrm{ZnCo}_{2} \mathrm{O}_{4}$. Cobalt oxide $\left(\mathrm{Co}_{3} \mathrm{O}_{4}\right)$ crystallizes in the normal cubic spinal structure $\mathrm{Co}^{2+}\left(\mathrm{Co}^{3+}\right)_{2}\left(\mathrm{O}^{2-}\right)_{4}$ (space group $\mathrm{O}_{\mathrm{h}}^{7}$ ) with $\mathrm{Co}^{2+}$ and $\mathrm{Co}^{3+}$ placed at tetrahedral (A-sites, magnetic moment $4.14 \mu_{B}$ ) and octahedral sites (B sites, diamagnetic), respectively $[51,52]$. AFM coupling of A-sites ions brings about the AFM ordered phase below Neel temperature (reported in $30-40 \mathrm{~K}$ range). It has been observed that in case of AFM nanoparticles the Neel temperature is reduced with respect to the bulk material and many new magnetic phenomena might appear (weak FM, spin canting, exchange bias effect) due to uncompensated surface or core spins.

An interesting study of magnetic properties of spinel $\mathrm{Co}_{3} \mathrm{O}_{4}(111)$ surface and its interface with $\mathrm{ZnO}$ (0001) has been reported by Kupchak et al. [53]. Spinel surface, containing $\mathrm{Co}^{2+}$ and $\mathrm{Co}^{3+}$ ions and terminated with either cobalt or oxygen ions were considered. Calculation has shown that $\mathrm{Co}^{3+}$ ions have non-zero magnetic moments at the surface and interface, leading to the FM ordering. Since some heavily Co-doped $\mathrm{ZnO}$ samples may contain $\mathrm{Co}_{3} \mathrm{O}_{4}$ as a secondary phase, such a magnetic ordering might be the possible origin of their magnetism.

A key phase that is formed during hydrothermal synthesis, $\mathrm{ZnCo}_{2} \mathrm{O}_{4}$, is a spinel that is known to be magnetic under certain oxygenation conditions [54]. It has a cubic structure, is a typical representative of normal $\mathrm{Zn}^{2+}\left(\mathrm{Co}^{3+}\right)_{2} \mathrm{O}_{4}$ spinel and belongs to the Fd3m space group with $Z=8$. In an ideal $A_{2} \mathrm{O}_{4}$ spinel structure, A atoms are located on tetrahedral sites of $T_{d}$ symmetry, while $B$ atoms are on octahedral sites of $D_{3 d}$ symmetry and oxygen atom occupy $\mathrm{C}_{3 \mathrm{v}}$ sites. In $\mathrm{ZnCo}_{2} \mathrm{O}_{4}$ the anions form a nearly ideal close-packed pseudo-face-center-cubic sublattice surrounded by tetrahedral and octahedral sites where cations occupy only $1 / 8$ of the tetrahedrally coordinated sites and $1 / 2$ of the octahedrally coordinated sites. $\mathrm{ZnCo}_{2} \mathrm{O}_{4}$ is a transparent, $\mathrm{p}$-type or $\mathrm{n}$-type $\mathrm{FM}$ semiconductor relevant to spintronics and wide-bandgap electronics. $\mathrm{ZnCo}_{2} \mathrm{O}_{4}$ can be both FM and AFM. AFM is realized by Co-O-Co superexchange, FM by Co-Co hole mediated exchange. For a large enough number of holes $\mathrm{ZnCo}_{2} \mathrm{O}_{4}$ can be FM.

\section{SAMPLES AND CHARACTERISATION}

Samples $n \mathrm{CoO} /(1-n) \mathrm{ZnO}(n=0.05,0.10,0.20,0.30$, $0.40,0.50,0.60,0.70$ ) were obtained using the hydrothermal method. Initially, a mixture of cobalt and zinc hydroxides was obtained by addition of $2 \mathrm{M}$ solution of $\mathrm{KOH}$ to the $20 \%$ solution of a proper amount of $\mathrm{Zn}\left(\mathrm{NO}_{3}\right) \cdot 6 \mathrm{H}_{2} \mathrm{O}$ and $\mathrm{Co}\left(\mathrm{NO}_{3}\right) \cdot 6 \mathrm{H}_{2} \mathrm{O}$ in water. Next, a mixture of the obtained hydroxides were put in the reactor heated with microwaves. The microwave assisted synthesis of all samples was conducted under a pressure of $3.8 \mathrm{MPa}$ for $15 \mathrm{~min}$. The synthesized product was filtered and dried. The morphology of samples was investigated using scanning electron microscope (SEM, Hitachi) followed by the phase composition of the samples determined by the X-ray diffraction (XRD, $\mathrm{Co}_{\mathrm{K \alpha}}$ radiation, $X$ 'Pert Philips). The specific surface area of the nanopowders was determined using the BrunauerEmmett-Teller (BET) method with the equipment Gemini 2360 of Micromeritics. The helium pycnometer AccuPyc 1330 of Micromeritics was applied to determine the density of powders.

Magnetization study was performed using a Quantum Design Magnetic Property Measurements System MPMS XL-7 with superconducting quantum interference device (SQUID) magnetometer in magnetic fields up to $70 \mathrm{kOe}$.

\section{RESULTS AND DISCUSSION}

According to the results of the XRD analysis, the $X R D$ spectra revealed the presence of $\mathrm{ZnO}, \mathrm{Co}(\mathrm{OH})_{2}$, and $\mathrm{ZnCo}_{2} \mathrm{O}_{4}$ phases. As expected, spinel phase $\mathrm{ZnCo}_{2} \mathrm{O}_{4}$ content increases with increasing $\mathrm{CoO}$ content, while the $\mathrm{ZnO}$ content decreases simultaneously. The mean crystallite size of the detected phases was determined using Scherrer formula. In particular, the mean crystallite size of $\mathrm{ZnFe}_{2} \mathrm{O}_{4}$ varied from 8 to $12 \mathrm{~nm}$. The investigations by SEM allowed to distinguish three different types of mor- 


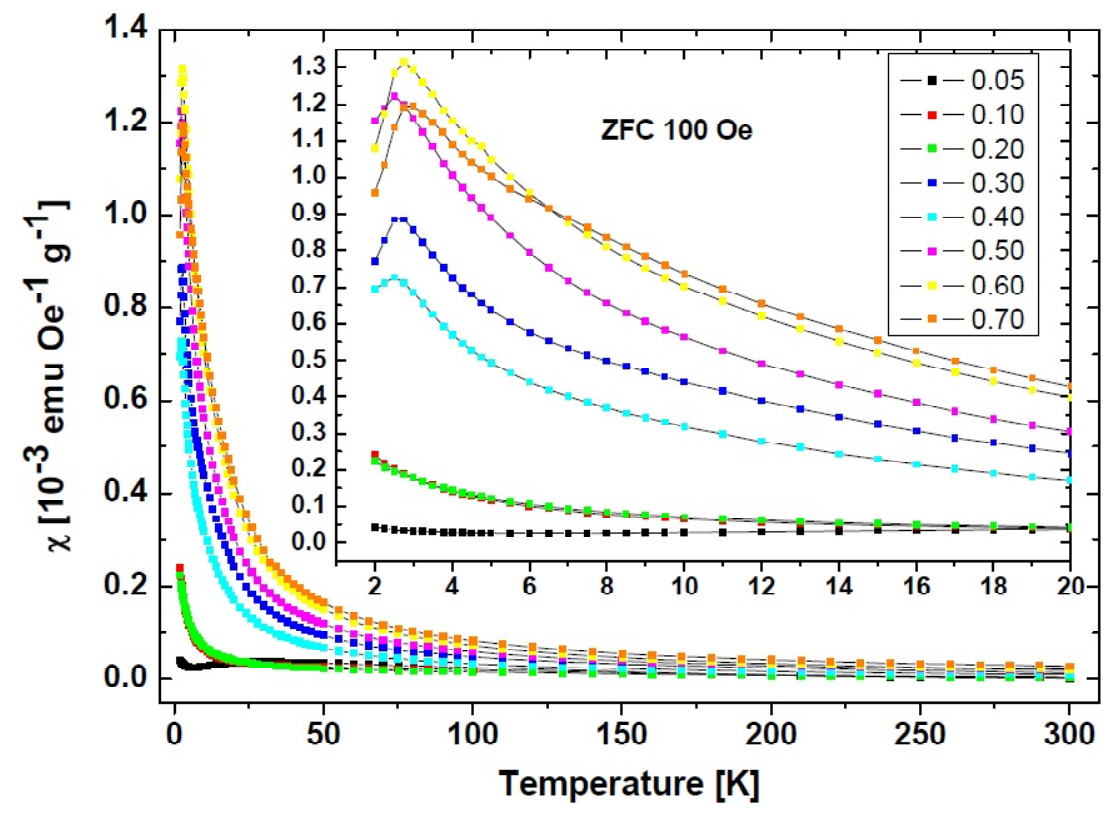

Fig. 1. Temperature dependence of the dc magnetic susceptibility $\chi(T)$ for all investigated samples in an external magnetic field $H=100$ Oe measured in ZFC mode. The insert shows magnified view of $\chi(T)$ curves in the low temperature range.

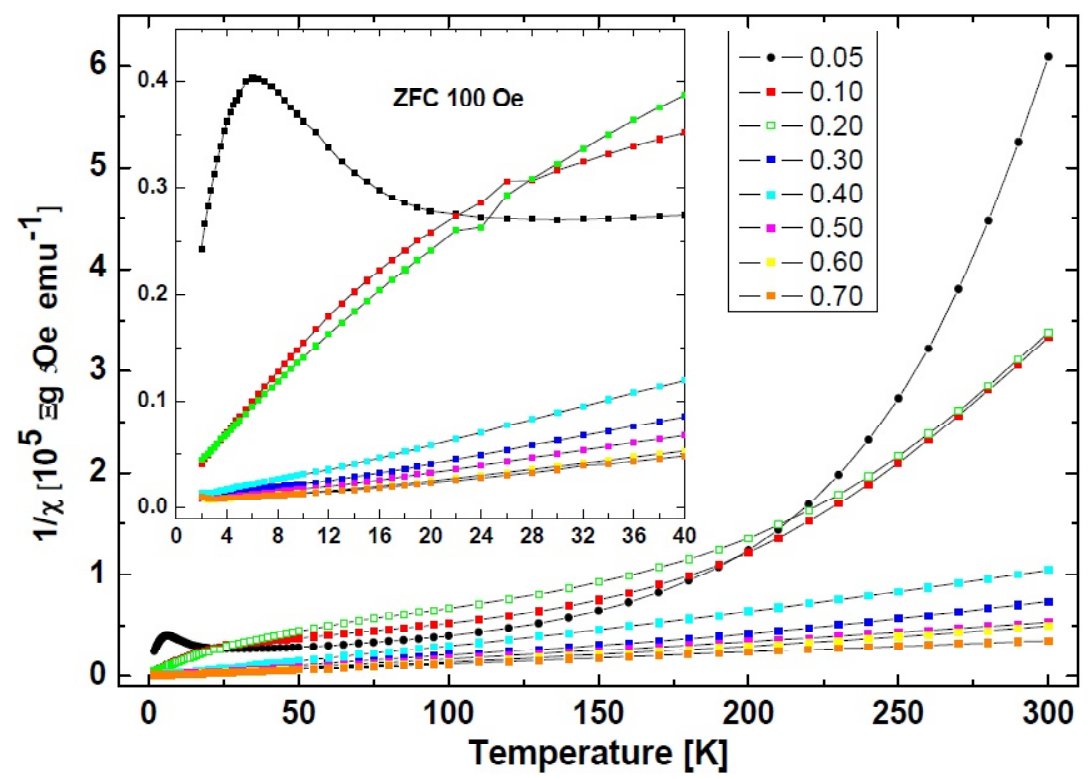

Fig. 2. Temperature dependence of the inverse magnetic susceptibility $\chi^{-1}(T)$ for all investigated samples in magnetic field $H=100$ Oe measured in ZFC mode. The insert shows magnified view of $\chi^{-1}(T)$ curves in the low temperature range.

phology: small spheroidal forms, large plates and the rods. The helium density and the specific surface area of samples were at a similar level. The low density of samples may be due to the presence of cobalt hydroxide, as confirmed by XRD analysis.

Fig. 1 shows the temperature dependence of $\mathrm{dc}$ magnetic susceptibility $\chi$ (defined as $\chi=M / H$ ) for all eight samples $(n=0.05,0.10,0.20,0.30,0.40$, $0.50,0.60,0.70)$ registered in an external magnetic field $H=100$ Oe in ZFC mode. The insert displays magnified view of $\chi(T)$ curves in the low temperature range. Except for the lowest temperatures, where a maximum in $\chi(T)$ is observed for certain samples $(n>0.20)$, the temperature dependence of dc susceptibility displayed in Fig. 1 shows a behavior typical for paramagnetic materials, with a strong increase of $\chi$ at lowest temperatures. To check if the Curie-Weiss law is fulfilled for our samples at high temperatures ( $T>50 \mathrm{~K})$, Fig. 2 presents the temperature dependence of the reciprocal susceptibil- 


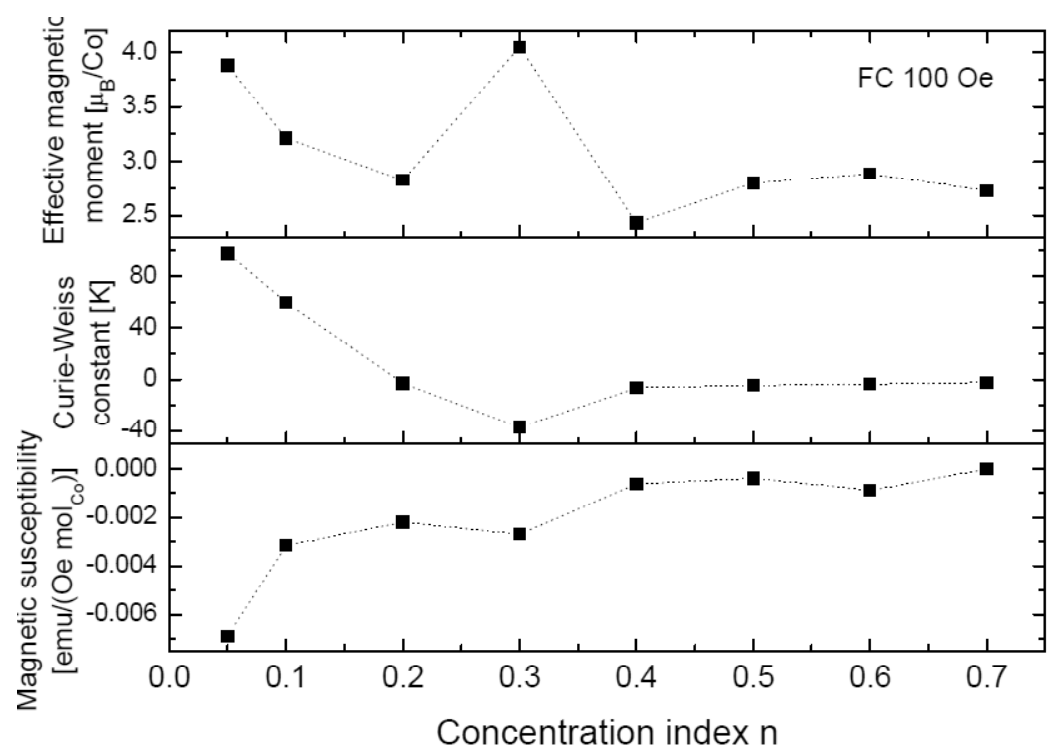

Fig. 3. Dependence of the effective magnetic moment $\mu_{\text {eff }}$ (top panel), the Curie-Weiss constant $T_{0}$ (middle panel), and temperature-independent susceptibility $\chi_{0}$ (bottom panel) on the concentration index $n$.

ity $\chi^{-1}(T)$. Because there is no broad temperature range were the experimental points are aligned along a straight line (especially for $n=0.05,0.10$, and 0.20 samples), an additional term in $\chi^{-1}(T)$ fitting has to been added that is independent on temperature. Thus, the fitting function used for description of the temperature dependence of dc susceptibility contains two terms:

$$
\chi(T)=\frac{c}{T-T_{0}}+\chi_{0},
$$

where $C$ is the Curie constant, $T_{0}$ is the Curie-Weiss temperature, and $\chi_{0}$ represents temperature independent susceptibility. Fitting of experimental data with function (1) gave very satisfactory results and the solid lines in Fig. 2 are the best least-squares fits. If the Curie constant is express in (emu.K/ molOe) unit (molar Curie constant $-C_{\text {mol }}$ ), the effective magnetic moment (in units of Bohr magnetons) for a single Co ion could be calculated:

$\mu_{\text {eff }}=\sqrt{\frac{3 k_{B} C_{\text {mol }}}{N_{A} \mu_{B}^{2}}} \cong 2.824 \sqrt{C_{\text {mol }}}$,

where $k_{B}$ is Boltzmann constant, $N_{A}$ is Avogadro constant, and $\mu_{B}$ Bohr magneton. The obtained values of the effective magnetic moment $\mu_{\text {eff }}$ the $\mathrm{Cu}$ rie-Weiss temperature $T_{0}$, and temperature-independent susceptibility $\chi_{0}$ for all investigated samples are presented in Fig. 3.

The effective magnetic moment in our samples varies in 2.5-4.0 $\mu_{B}$ /Co range for different samples at high temperatures. It is slightly smaller than ex- pected for the high-spin $\mathrm{Co}^{2+}$ ion $(S=3 / 2, g=2.2, \mu$ $\sim 4.2 \mu_{B}$ ). This could be the result of AFM paring or clustering of cobalt ions producing magnetic entities with a magnetic moment equal to zero. The Curie-Weiss temperature $T_{0}$ carries the information about the strength of the interaction between paramagnetic species and the type of that interaction (FM for positive, AFM for negative $T_{0}$ values). This parameter is positive for $n=0.05$ and 0.10 samples what suggests FM interaction between $\mathrm{Co}$ ions and its strength seems to decrease with increase of Co contents for lightly doped samples. On the other hand, for $n=0.30$ sample $T_{0}$ is negative (AFM interaction) and for the rest of our samples close to zero, what indicates negligible interactions.

Temperature-independent susceptibility $\chi_{0}$ has a negative value (diamagnetic) for all our samples and its value increases to zero with the increase of Co concentration (Fig. 3, bottom panel). It suggests the decrease of the diamagnetic contribution in heavily doped $n \mathrm{CoO} /(1-n) \mathrm{ZnO}$ nanocomposites. On the other hand the value of $\chi_{0}$ for different samples depends on the strength of external magnetic field (see Fig. 4). For strong magnetic fields (of about $70 \mathrm{kOe}$ ) the values of $\chi_{0}$ for all samples appear to converge to a common, small negative value (of about -0.001 $\mathrm{emu} /(\mathrm{Oe} \cdot \mathrm{mole} \mathrm{Co})$ ). It is interesting to note that for samples with smaller concentration of Co ions $(n=$ $0.05,0.10,0.20,0.30$ ) the value of $\chi_{0}$ increases with magnetic field, while for samples with higher concentration of cobalt ions $(n=0.40,0.50,0.60,0.70)$ this dependence is reversed. Apparently, cobalt concentration in our samples has affected directly 


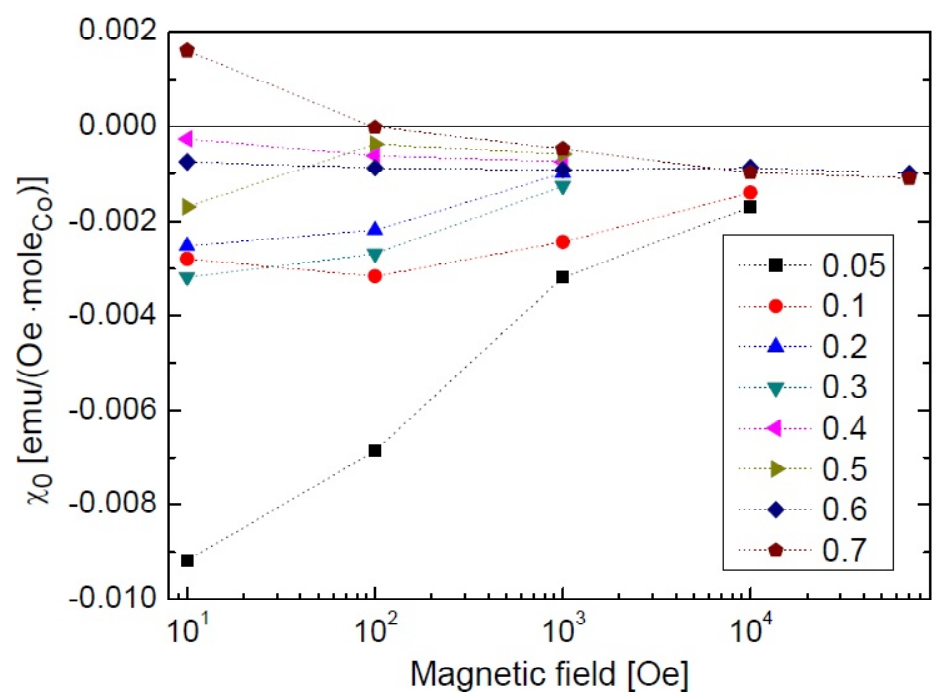

Fig. 4. Dependence of temperature-independent susceptibility $\chi_{0}$ on an external magnetic field in the investigated samples.

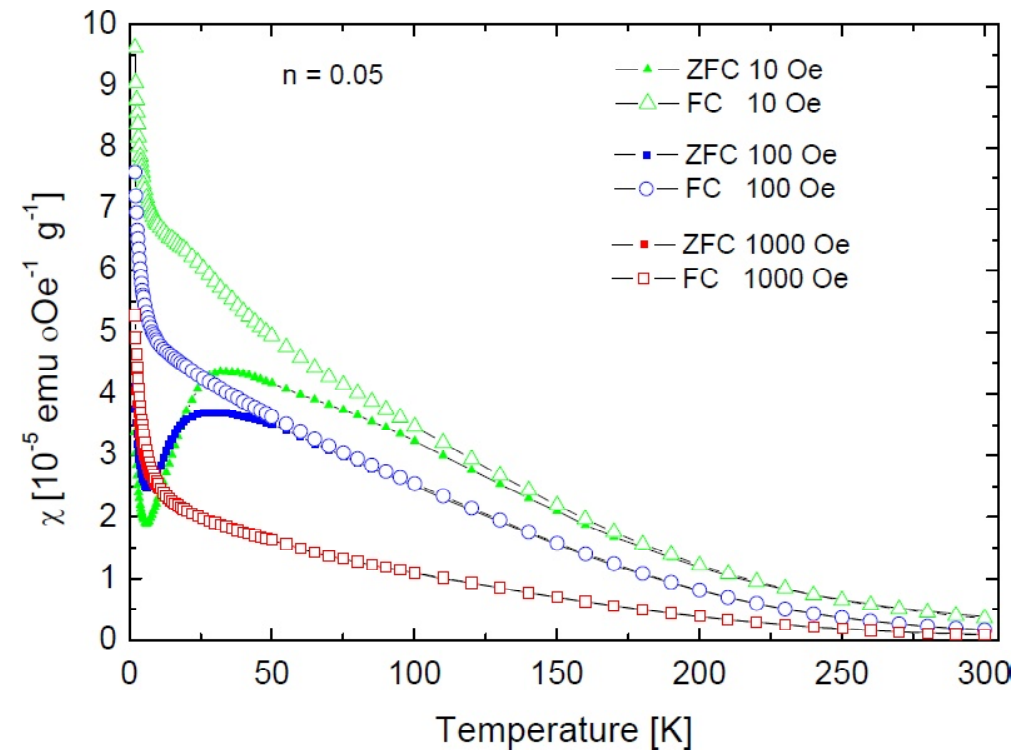

Fig. 5. Temperature dependence of the dc magnetic susceptibility $\chi(T)$ for $n=0.05$ sample in ZFC and FC modes in external magnetic fields $H=10,100$, and $1000 \mathrm{Oe}$.

the magnetic feature related to the diamagnetic component.

Fig. 5 presents the temperature dependence of the dc magnetic susceptibility $\chi(T)$ for $n=0.05$ sample in ZFC and FC modes in three external magnetic fields: $H=10,100$, and 1000 Oe. This particular sample shows a unique magnetic behaviour in $30-35 \mathrm{~K}$ range in weak magnetic fields in form of a local maximum in $\chi(T)$ curve measured in ZFC mode. This maximum is easily visible in magnetic fields $H$ $=10$ and $100 \mathrm{Oe}$, but in $H=1000$ Oe both $\chi_{Z F C}(T)$ and $\chi_{F C}(T)$ curves coincide and no local maximum is evident in that range. Considering similarity of the temperature of this local maximum in $n=0.05$ sample and the Neel temperature $T_{N}$ of $\mathrm{Co}_{3} \mathrm{O}_{4}$ spinel phase ( $T_{N}=33 \mathrm{~K}$ for bulk cobalt oxide) it is tempting to attribute the observed local maximum to the presence of that particular secondary phase. Although $\mathrm{Co}_{3} \mathrm{O}_{4}$ nanoparticles were not detected in hydrothermal samples by X-ray diffraction (probably due to their low concentration and limited sensitivity of that method), their presence was observed by a more sensitive Electron Paramagnetic Resonance technique [17].

Another interesting feature that appears only in the very low temperature range $(T<3 \mathrm{~K})$ is a maximum in $\chi_{Z F C}(T)$ curve in samples with not so low concentration of cobalt ions (i.e. samples $n=0.30$, $0.40,0.50,0.60,070$ ) (see insert in Fig. 1), while no local maximum in $\chi_{F C}(T)$ curves is observed. Such 

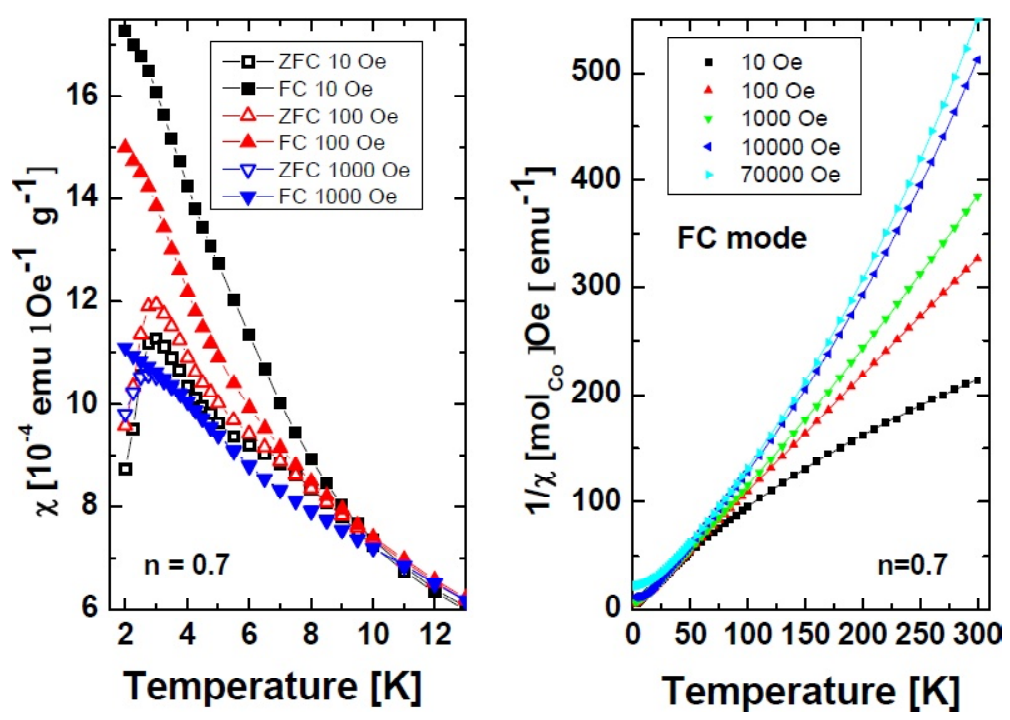

Fig. 6. (Left panel) Temperature dependence of the dc magnetic susceptibility $\chi(T)$ in the low temperature range for $n=0.70$ sample in ZFC and FC modes in external magnetic fields $H=10,100$, and $1000 \mathrm{Oe}$. (Right panel) Temperature dependence of the inverse magnetic susceptibility $\chi^{-1}(T)$ for $n=0.70$ samples in FC mode in five different external magnetic fields.

a behaviour of magnetic susceptibilities in ZFC and FC modes is usually attributed to the presence of the superparamagnetic nanoparticles, in our case to $\mathrm{ZnCo}_{2} \mathrm{O}_{4}$ nanoparticles. The temperature at which a maximum of $\chi_{Z F C}(T)$ curve is registered is called the blocking temperature $T_{B}$ and it separates the superparamagnetic state $\left(T>T_{B}\right)$ and blocked state $\left(T<T_{B}\right)$ of magnetic nanoparticles. Often a simple relation between magnetocrystalline and thermal energy is used to estimate the blocking temperature, $K V=25 k T_{B}$, where $K$ is magnetocrystalline anisotropy energy density constant, $V$ the volume of a nanoparticle, and $k$ is Boltzmann's constant. Above $T_{B}$ the Zeeman energy $\mu \cdot H$ (where $\mu$ is the magnetic moment and $H$ an external magnetic field) is smaller than the thermal energy what causes randomization of magnetic moments and the net magnetisation decreases with the increase of temperature (superparamagnetic phase).

Fig. 6 illustrates the separation of $\chi_{Z F C}(T)$ and $\chi_{F C}(T)$ curves and a maximum in $\chi_{Z F C}(T)$ curve for a specific sample $n=0.70$. The value of $T_{B}$ varies slightly for different samples (dependence on the composition index $n$ ) and depends on the strength of external magnetic field $\mathrm{H}$. Fig. 7 shows this dependence for four samples with higher concentration of $\mathrm{CoO}$ in an initial mixture (samples $n=0.40$, $0.50,0.60,0.70)$. As is typical for magnetic nanoparticles, $T_{B}$ decreases with the increase of the magnetic field strength. This is because the external magnetic field lowers the barier between two easy axis orientations of a magnetic nanoparticle. It is also interesting to notice that $T_{B}$ increases with the composition index $n$ and thus with the cobalt concentration. Such dependence can be explained by an increse of an average size of $\mathrm{ZnCo}_{2} \mathrm{O}_{4}$ nanoparticles with increasing Co concentration.

In Fig. 8 the isothermal (at $T=2 \mathrm{~K}$ ) magnetisation of all investigated samples in magnetic fields up to $70 \mathrm{kOe}$ is presented. As expected for magnetic nanoparticles, magnetisation in the form of a hysteresis loop is observed because at that temperature $\left(T<T_{B}\right)$ our samples are in the blocked, FM state. On the other hand, at RT practically no hysteresis loop is registered because this temperature is much higher than the blocking temperature and the samples are in superparamagnetic state (Fig. 9). Thus no RTFM is registered in our samples. The values of two parameters of the observed hysteresis loops, the remanent magnetisation $M_{r}$ and the coercive field $H_{c}$, at $T=2 \mathrm{~K}$ are presented in graphical form in Fig. 10. The values of the coercive field, with the exception of $n=0.05$ sample, are in 10 - 100 Oe range and increase with the increase of Co concentration. For $n=0.05$ sample a much higher value $H_{c}=170 \mathrm{Oe}$ is obtained and it do not obey the $H_{c}(n)$ dependence observed for other samples, probably due to different magnetic phases contents. In that sample there is not enough cobalt to form significant amount of $\mathrm{ZnCo}_{2} \mathrm{O}_{4}$ nanoparticles and almoust all Co enter substitutionally into $\mathrm{ZnO}$ phase. Besides, the $\mathrm{Co}_{3} \mathrm{O}_{4}$ phase is also detected in that sample. The remanent magnetization $M_{r}$ of 


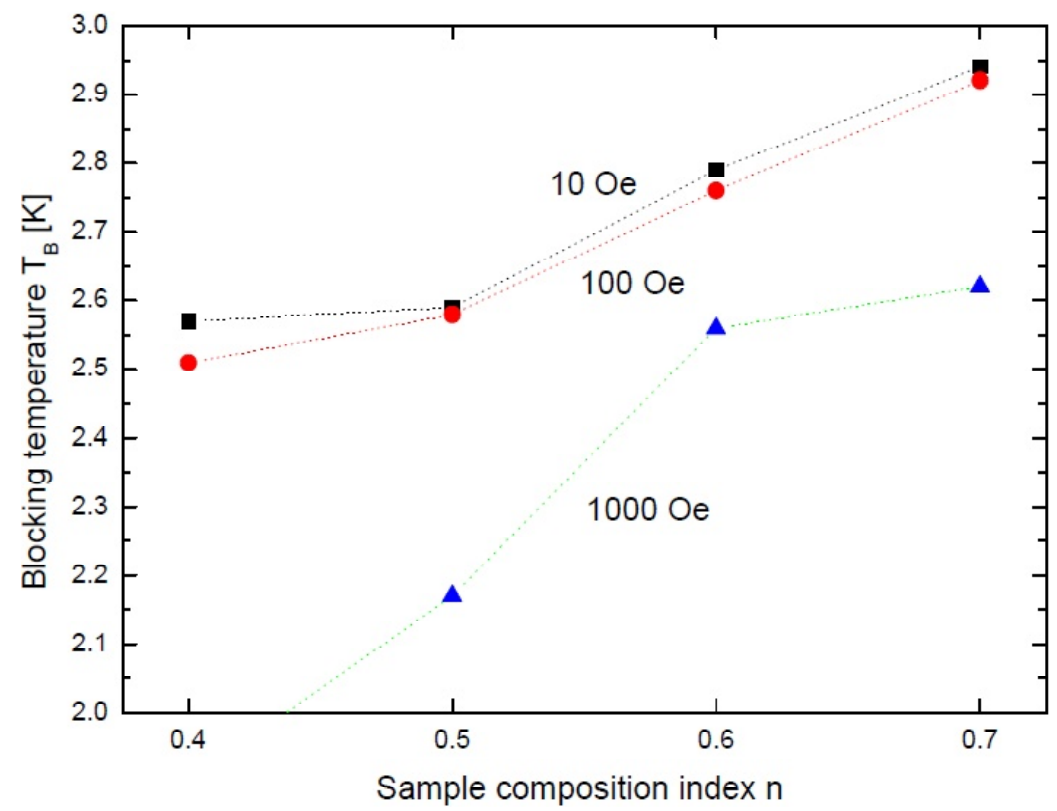

Fig. 7. Dependence of the blocking temperature $T_{B}$ on external magnetic field $H(H=10,100,1000 \mathrm{Oe})$ in four samples $(n=0.40,0.50,0.60,0.70)$ synthesised under pressure 3.8 MPa. Blocking temperature for sample $n=0.30$ in $H=1000$ Oe is below $2 \mathrm{~K}$ and could not be measured on our magnetometer.

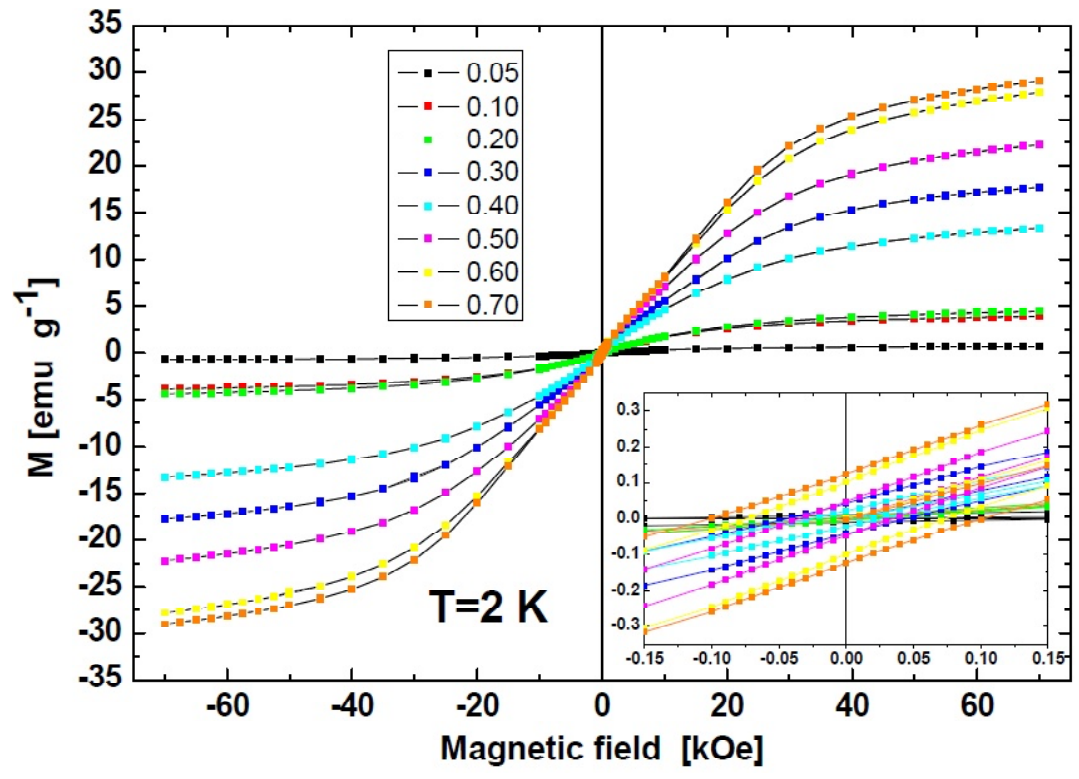

Fig. 8. Isothermal magnetisation $M(H)($ at $T=2 \mathrm{~K})$ for all investigated samples. The insert shows magnified central part of hysteresis loops.

the studied samples is in $0.005-0.125 \mathrm{emu} / \mathrm{g}$ range and follows roughly the same simple rule as the coercive field does - the higher the Co concentration, the higher the remanent magnetization.

The saturation magnetization $M_{s}$ is not so straightforward to obtain from the registered loops because the applied external magnetic field was not strong enough to ensure saturation. To estimate $M_{s}$ the following procedure was used: magnetization of samples measured in strong magnetic fields was presented as a function of reciprocal magnetic field strength and the intercept of the fitted straight line with magnetization axis was assume to be $M_{s}$ (see Fig. 11. left panel). The value of $M_{s}$ of different samples increases with the Co contents, what is to be expected, as this is the only magnetic ion in our samples. The obtained values of $M_{s}$ where used to calculate the effective magnetic moment per one Co ion in our samples (Fig. 11, right panel). It will be instructive to compare this plot of the effective magnetic moment as a function of the $\mathrm{CoO}$ composition index $n$ that was calculated from the hysteresis 


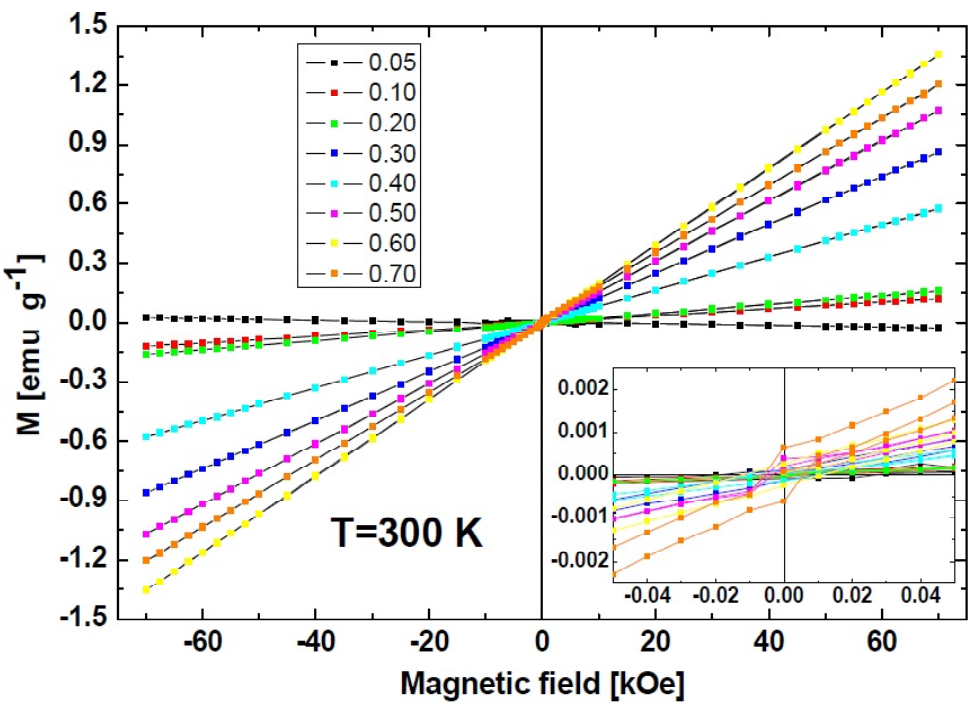

Fig. 9. Isothermal magnetisation $M(H)$ (at $T=300 \mathrm{~K})$ for all investigated samples. The insert shows magnified central part of hysteresis loops.

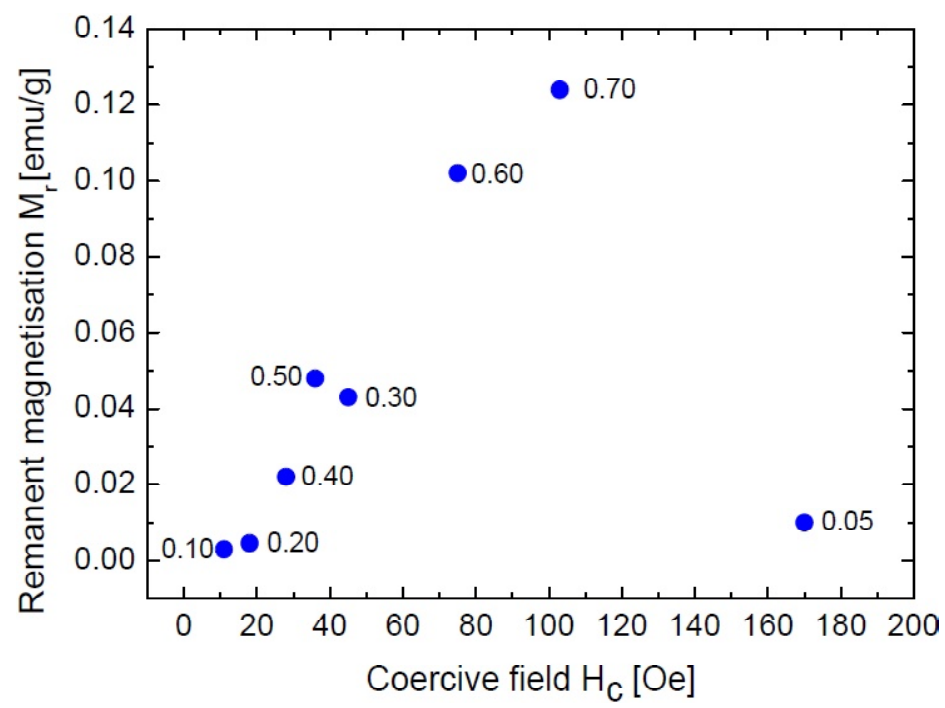

Fig. 10. Graphical presentation of magnetic loop parameters (remanent magnetisation, coercive field) determined at $T=2 \mathrm{~K}$ for all studied samples.

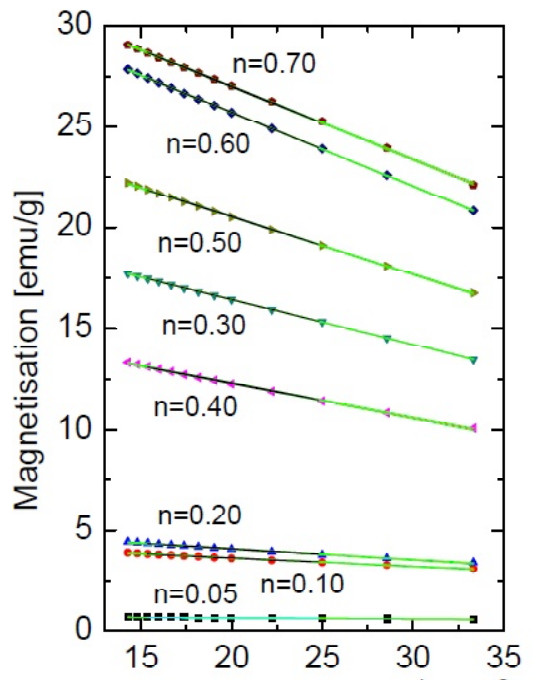

Reciprocal magnetic field $\mathrm{H}^{-1}\left[10^{-6} \mathrm{Oe}^{-1}\right]$

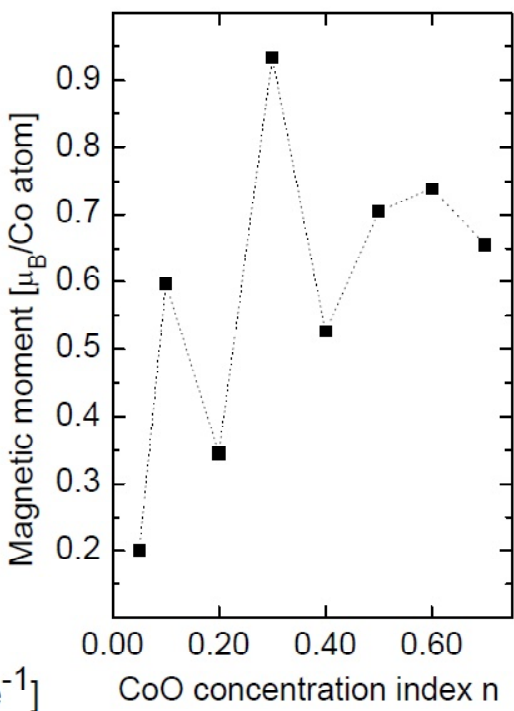

$\mathrm{CoO}$ concentration index $\mathrm{n}$

Fig. 11. (Left panel) Dependence of magnetisation on reciprocal magnetic field $M\left(H^{-1}\right)$ obtained in strong $(H>30 \mathrm{kOe})$ fields for all samples. (Right panel) Calculated effective magnetic moment of a single Co ion in samples with different composition index $n$. 
loops measurements made at $T=2 \mathrm{~K}$ with a similar plot obtained from the Curie-Weiss law in the hightemperature range (Fig. 3, top panel). Except for $n$ $=0.05$ sample, both plots display a very similar form, but the values of the magnetic moment of a single Co ion at $T=2 \mathrm{~K}$ are significantly reduced (roughly four times) when compared with the high-temperature values. This could be explained by formation of many AFM pairs or clusters at low temperatures that do not contribute to an effective magnetic moment and thus decrease an average moment calculated for a single Co ion. Sample $n=0.05$ do not follow the overall pattern because its phase content is different from the rest of samples. This dissimilarity of $n=0.05$ sample was already been commented during discussion of the hysteresis loop parameters. Generally, the effective magnetic moment of a single Co ion seems to increase with Co concentration up to $n=0.60$ (with a possible exception of $n=0.30$ sample) and then slowly decreases with further increase of the concentration index $n$. This behaviour can be easily correlated with the relative abundance of $\mathrm{ZnCo}_{2} \mathrm{O}_{4}$ phase in our samples, which reaches a maximum for $n_{0}=0.65$ (for $n$ $<n_{0}$ there is not enough Co to form large amount of $\mathrm{ZnCo}_{2} \mathrm{O}_{4}$, for $n>n_{0}$ there is deficit of $\mathrm{Zn}$ which limits formation of $\mathrm{ZnCo}_{2} \mathrm{O}_{4}$ phase). Thus the obtained dependence of the effective magnetic moment of a single $\mathrm{Co}$ ion on composition index $n$ demonstrates that $\mathrm{ZnCo}_{2} \mathrm{O}_{4}$ nanoparticles provide the most important contribution to the magnetism of $n \mathrm{CoO} /(1-n) \mathrm{ZnO}$ nanocomposites.

\section{CONCLUSIONS}

Magnetometric study of a wide range of $n \mathrm{CoO} /(1-$ n) $\mathrm{ZnO}$ nanocomposites (with the composition index $n$ ranging from 0.05 up to 0.70 ) synthesized by hydrothermal method revealed the absence of RTFM in all our samples. The main reason why FM signal was discovered only in the low temperature range seems to be the magnetic inhomogeneity of the investigated samples which stems from the presence of different phases $\left(\mathrm{Zno}, \mathrm{CoO}, \mathrm{Co}_{3} \mathrm{O}_{4}, \mathrm{ZnCo}_{2} \mathrm{O}_{4}\right.$, $\left.\mathrm{Co}(\mathrm{OH})_{2}\right)$ and various structural defects. Sample $n$ $=0.05$ differs significantly from the rest of the studied $n \mathrm{CoO} /(1-n) \mathrm{ZnO}$ nanocomposites as most of Co ions there enters substitutionally into $\mathrm{ZnO}$ matrix, and the rest of cobalt ions is involved in the formation of small amounts of $\mathrm{Co}_{3} \mathrm{O}_{4}$ and $\mathrm{ZnCo}_{2} \mathrm{O}_{4}$ phases. In nanocomposites with the composition index $n \geq 0.10$ the most important role - as magnetic properties are concerned - play the $\mathrm{ZnCo}_{2} \mathrm{O}_{4}$ nanoparticles. The superparamagnetic state of these nanomaterials spans a broad temperature range, starting at the low blocking temperature of $\sim 2.5 \mathrm{~K}$. The effective magnetic moment of a single $\mathrm{Co}$ ion follows the contents of $\mathrm{ZnCo}_{2} \mathrm{O}_{4}$ phase but is also strongly temperature dependent. In the low temperature range many Co ions are involved in AFM pairs and clusters and do not contribute to the overall magnetism of $n \mathrm{CoO} /(1-n) \mathrm{ZnO}$ nanocomposites.

\section{REFERENCES}

[1] Z. L. Wang // J. Phys.: Condens. Mat. 16 (2004) R829.

[2] A. Janotti and Ch.G. Van de Walle // Rep. Prog. Phys. 72 (2009) 126501.

[3] A. Kolodziejczak-Radzimska and T. Jesionowski // Materials 7 (2014) 2833.

[4] A. K. Sood, Z. L. Wang, D. L. Polla, N. K. Dhar, T. Manzur and A.F.M. Anwar, In: Optoelectronic Devices and Properties, ed. by Oleg Sergiyenko (In Tech, 2011), p. 173.

[5] X. Chen, Z. Wu, D. Liu and Z. Gao // Nanoscale Res. Lett. 12 (2017) 143.

[6] Z. L. Wang and J. Song // Science 312 (2006) 243.

[7] M. Vaseem, A. Umar and Y.-B. Hahn, In: Metal oxide nanostructures and their applications, vol. 5, ed. by Ahmad Umar and Yoon-Bong Hahn (American Scientific Publishers, 2010), p. 1.

[8] A. K. Arora, S. Devi, V. S. Jaswal, J. Singh, M. Kinger and V. D. Gupta // Oriental J. Chemistry 30 (2014) 1671.

[9] K. Prasad and A. K. Jha // Natural Science 1 (2009) 129.

[10] A. N. UI Haq, A. Nadhman, I. Ullah, G. Mustafa, M. Yasinzai, and I. Khan // Journal of Nanomaterials 2017 (2017) 8510342.

[11] T. Dietl, H. Ohno, F. Matsukura, J. Cibert, and D. Ferrand // Science 287 (2000) 1019.

[12] J. M. D. Coey, P. Stamenov, R. D. Gunning, M. Venkatesan, and K. Paul // New J. Phys. 12 (2010) 053025.

[13] Y. Tian, Y. Li, M. He, I. A. Putra, H. Peng, B. Yao, S. A. Cheong, and T. Wu // Appl. Phys. Lett. 98 (2011) 162503.

[14] A. Bonanni, T. Dietl // Chem. Soc. Rev. 39 (2010) 528.

[15] K. Ueda, H. Tabata and T. Kawai // Appl. Phys. Lett. 79 (2001) 988.

[16] H. L. Tao, Z. H. Zhang, L. L. Pan, M. He, B. Song and Q. Li // Int. J. Mod. Phys. B 27 (2013) 1350078. 
[17] J. Typek, N. Guskos, G. Zolnierkiewicz, D. Sibera and U. Narkiewicz // Rev. Adv. Mater. Sci. 50 (2017) 76.

[18] K. Samanta, P. Bhattacharya, R.S. Katiyar, W. Iwamoto, P.G. Pagliuso and C. Rettori // Phys. Rev. B73 (2006) 245213.

[19] J. H. Kim, H. Kim, D. Kim, Y. E. Ihm and W. K. Choo // J. Appl. Phys. 92 (2002) 6066.

[20] J. S. Thakur, G. W. Auner, V.M. Naik, C. Sudakar, P. Kharel, G. Lawes, R. Suryanarayanan and R. Naik // J. Appl. Phys. 102 (2007) 093904.

[21] H. Harima // J. Phys.: Condens. Mat. 16 (2004) S5653.

[22] J. Park, M. G. Kim, H. M. Jang, S. Ryu and Y. M. Kim // Appl. Phys. Lett. 84 (2004) 1338.

[23] B. B. Straumal, A. A. Mazilkin, S. G. Protasova, P. B. Straumal, A. A. Myatiev, G. Schütz, E. Goering and B. Baretzky // Phys. Metal. Metallurgy 113 (2012) 1244.

[24] J. M. D. Coey // Solid State Sci. 7 (2005) 660.

[25] T. Tietze, P. Audehm, Y-Ch. Chen, G. Schutz, B. B. Straumal S. G. Protasova, A. A. Mazilkin, P. B. Straumal, T. Prokscha, H. Luetkens, Z. Salman, A. Suter, B. Baretzky, K. Fink, W. Wenzel, D. Danilov and E. Goering // Sci. Reports 5 (2015) 8871.

[26] X. Zhang, W. Zhang, X. Zhang, X. Xu, F. Meng and C. C. Tang // Adv, Cond. Mater. Phys. 2014 (2014) 806327.

[27] M. A. Garcia, J. M. Merino and E. F. Pinel // Nano Letters 7 (2007) 1489.

[28] T. Dietl, H. Ohno, F. Matsukura, J. Cibert and D. Ferrand // Science 287 (2000) 1019.

[29] K. Sato and H. Katayama-Yoshida // Semicond. Sci. Technol. 17 (2002) 367.

[30] A. Walsh, J. L. F. Da Silva, and S-H. Wei // Phys. Rev. Lett. 100 (2008) 256401.

[31] C. D. Pemmaraju, R. Hanafin, T. Archer, H. B. Braun and S. Sanvito // Phys. Rev. B 78 (2008) 054428.

[32] K. C. Verma, R. Bhatia, S. Kumar and R. K. Kotnala // Mat. Res. Express 3 (2016) 076103.

[33] J. M. D. Coey, M. Venkatesan and V. B. Fitzgerald // Nature Mater. 4 (2005) 173.

[34] R. Hanafin, C.D. Pemmaraju, T. Archer and S. Sanvito // Acta Phys. Pol. A 115 (2009) 263.
[35] V. Ney, S. Ye, K. Ollefs, T. Kammermeier, F. Wilhelm, A. Rogalev and A. Ney // J. Nanosci. Nanotechn. 10 (2010) 5958.

[36] R. O. Kuzian, J. Richter, M. D. Kuzmin and R. Hayn // Phys. Rev. B 93 (2016) 214433

[37] L. R. Shah, H. Zhu, W. G. Wang, B. Ali, T. Zhu, X. Fan, Y. Q. Song, Q. Y. Wen, H. W. Zhang, S. I. Shah and J. Q. Xiao // J. Phys. D: Appl. Phys. 43 (2010) 035002.

[38] T. Shi, Z. Xiao, Z. Yin, X. Li, Y. Wang, H. He, J. Wang, W. Yan and S. Wei // Appl. Phys. Lett. 96 (2010) 211905.

[39] D. A. Schwartz and D. R. Gamelin // $A d v$. Mater. 16 (2004) 2115.

[40] M. Sawicki, E. Guziewicz, M. I. Łukasiewicz, O. Proselkov, I. A. Kowalik, W. Lisowski, P. Dluzewski, A. Wittlin, M. Jaworski, A. Wolska, W. Paszkowicz, R. Jakiela, B. S. Witkowski, L. Wachnicki, M. T. Klepka, F. J. Luque, D. Arvanitis, J. W. Sobczak, M. Krawczyk, A. Jablonski, W. Stefanowicz, D. Sztenkiel, M. Godlewski, and T. Dietl // Phys. Rev. B 88 (2013) 085204.

[41] A. Ney, M. Opel, T. C. Kaspar, V. Ney, K. Ollefs, T. Kammermeier, S. Bauer, K.-W. Nielsen, S. T. B. Goennenwein, M. H. Engelhard, S. Zhou, K. Potzger, J. Simon, W. Mader, S. M. Heald, J. C. Cezar, F. Wilhelm, A. Rogalev, R. Gross and S. A. Chambers // New. J. Phys. 12 (2010) 013020.

[42] M. Opel, K.-W. Nielsen ,S. Bauer, S. T. B. Goennenwein, , J. C. Cezar, D. Schmeisser, J. Simon, W. Mader and R. Gross // Eur. Phys. J. B 63 (2008) 437.

[43] S. Zhou, K. Potzger, J. von Borany, R. Grötzschel, W. Skorupa, M. Helm and J. Fassbender // Phys. Rev. B 77 (2008) 035209.

[44] T. C. Kaspar, T. Droubay, S. M. Heald, M. H. Engelhard, P. Nachimuthu and S. A. Chambers // Phys. Rev. B 77 (2008) 201303(R).

[45] T. Dietl, T. Andrearczyk, A. Lipinska, M. Kiecana, M. Tay and Y. Wu // Phys. Rev. B76 (2007) 155312.

[46] J. Blasco, F. Bartolome, L. M. Garcýa and J. Garcýa // J. Mater. Chem. 16 (2006) 2282.

[47] I. Kuryliszyn-Kudelska, B. Hadzic, D. Sibera, M. Romcevic, N. Romcevic, U. Narkiewicz, W. Łojkowski, M. Arciszewska and W. Dobrowolski // J. Alloys Comp. 561 (2013) 247. 
[48] I. Kuryliszyn-Kudelska, W. Dobrowolski, M. Arciszewska, N. Romčević, M. Romčević, B. Hadžić, D. Sibera, U. Narkiewicz and W. Lojkowski // Sci. Sintering 45 (2013) 31.

[49] B. Hadžić, N. Romčević, M. Romčević, I. Kuryliszyn-Kudelska, W. D. Dobrowolski, U. Narkiewicz and D. Sibera // Hem. Ind. 67 (2013) 695.

[50] B. Hadžić, N. Romcevic, M. Romcevic, I. Kuryliszyn-Kudelska, W. Dobrowolski, J. Trajic, D. Timotijevic, U. Narkiewicz and D. Sibera // J. Alloys Comp. 540 (2012) 49.
[51] W. L. Roth // J. Phys. Chem. Solids 25 (1964) 1.

[52] A. H. Hill, A. Harrison, C. Ritter, W. Yue and W. Zhou // J. Magn. Magn. Matter. 323 (2011) 226.

[53] I. M. Kupchak, N. F. Serpak, A. Chkrebtii and R. Hayn // arXiv:1704.07148 [cond-mat.mtrlsci]

[54] H. J. Kim, I. C. Song, J. H. Sim, H. Kim, D. Kim, Y. E. Inm and W. K. C. Choo // J. Appl. Phys. 95 (2004) 7387. 